\title{
Evolution in Understanding of Cardiovascular Outcomes in Diabetes
}

\author{
Anuj Maheshwari \\ Department of General Medicine, BBD University, Lucknow, India \\ Email: dranujm@gmail.com
}

How to cite this paper: Maheshwari, A. (2021) Evolution in Understanding of Cardiovascular Outcomes in Diabetes. Journal of Diabetes Mellitus, 11, 378-387. https://doi.org/10.4236/jdm.2021.115030

Received: October 23, 2021

Accepted: November 15, 2021

Published: November 18, 2021

Copyright $\odot 2021$ by author(s) and Scientific Research Publishing Inc. This work is licensed under the Creative Commons Attribution International License (CC BY 4.0).

http://creativecommons.org/licenses/by/4.0/

\section{(c) (i) Open Access}

\begin{abstract}
Eighty percent mortality occurs in patients with type 2 diabetes mellitus due to cardiovascular (CV) events like myocardial infarction, stroke and peripheral vascular disease. We learned from United Kingdom Prospective Diabetes Study (UKPDS) that lowering HbAlc reduces both micro and macrovascular complications. However, more recent studies have provided insight that $\mathrm{HbA} 1 \mathrm{c}$ reduction may exert only a modest effect in lowering macrovascular complications. In fact, treating hypertension and dyslipidemia may provide a greater benefit in reducing CV events. The last 10 years have changed our understanding of cardiovascular disease (CVD) risk in type 2 diabetes. Earlier it was believed to be due to atherosclerotic cardiovascular disease which was exaggerated by hyperglycemia. Additionally, some antidiabetic medications were also implied to contribute to cardiovascular illness and mortality. It is plausible that insulin resistance may play a significant role in cardiovascular complications as well since it induces endothelial dysfunction much earlier before type 2 diabetes is diagnosed. Many patients manifest CVD in form of either atherosclerotic cardiovascular disease (ASCVD) or heart failure prior to diagnosis of diabetes. Amelioration of insulin resistance may be the management key in type 2 diabetes and can be very well achieved with modern drugs although attaining and maintaining desirable glycemic control may be as crucial as noted in type 1 diabetes.
\end{abstract}

\section{Keywords}

Type 2 Diabetes Mellitus, CVD, Hyperglycemia, ASCVD, Heart Failure

\section{Introduction}

Cardiovascular disease (CVD) is a major contributor to morbidity and mortality among patients with diabetes. People with type 2 diabetes mellitus (T2DM) have twice the risk of manifesting cardiovascular ailments including myocardial in- 
farction (MI), stroke and peripheral vascular disease which account for eighty percent mortality [1]. A population-based study has demonstrated that cardiovascular deaths occur 7.5 times more in patients with type 2 diabetes who never had a myocardial infarction compared to people who never had diabetes or myocardial infarction [2]. 52.9\% mortality due to cardiovascular disease has been suggested in patients with diabetes by Chennai Urban Population Study which was significantly higher $(\mathrm{P}=0.042)$ than those who did not have diabetes $(24.2 \%)$. One year mortality rate after myocardial infarction was $44 \%$ in males and $36 \%$ in females, while it was $32 \%$ in males not having diabetes and $20 \%$ in non-diabetic females [3]. Apart from this, ten-year risk of developing coronary heart disease in patients having diabetes is reported to be more than 20 percent in comparison to non-diabetic population [4]. Considering these facts, Adult Treatment Panel III of the National Cholesterol Education Program has declared type 2 diabetes mellitus as coronary heart disease risk equivalent [5].

Many clinical trials have proven that $\mathrm{HbA1c}$ reduction in type 2 diabetes has no [6] or only a modest [7] [8] effect on reducing cardiovascular (CV) risk which is certainly not more than what is achieved by amelioration of hypertension and dyslipidemia. Intensive glycemic control of diabetes substantially reduces the microvascular complications but not the occurrence of macrovascular events like myocardial infarction or strokes. Stringent glucose control from the onset of the diagnosis certainly reduces macrovascular events and all-cause mortality later in life, "legacy effect" [9]. Lipid lowering and control of hypertension also play a key role in the primary prevention of cardiac risk in patients with type 2 diabetes mellitus [10]. It adds clinically significant reduction in deaths and manifestations due to cardiovascular causes. Tight blood pressure control significantly reduced the risk of any diabetes related end point by $24 \%(\mathrm{P}=0.0046)$, mortality by $32 \%$ $(\mathrm{P}=0.019)$, stroke by $44 \%$, microvascular end points by $37 \%(\mathrm{P}=0.013)$ and heart failure by $56 \%(\mathrm{P}=0.0043)$ [11]. Hyperglycemia is not always the culprit to cause cardiovascular adverse outcomes. Instead, hypoglycemia or certain antidiabetic therapies may contribute to adverse $\mathrm{CV}$ outcomes.

\section{Hypoglycemia: Does It Induce CVD?}

Hypoglycemia induces many indirect changes including inflammatory cytokine secretion, endothelial dysfunction, exacerbated coagulation, and fibrinolysis with potential of promoting adverse effects on cardiovascular morbidity and mortality [12]. Acute hypoglycemia induces significant sympatho-adrenal responses, because of enhanced autonomic activity with increased adrenaline secretion. It provokes hemodynamic changes to increase heart rate and peripheral systolic blood pressure, fall in central blood pressure, lowering of peripheral arterial resistance, increase in myocardial contractility, stroke volume and cardiac output. These changes lead to the development of transient, but markedly increased workload on the heart, which may have adverse outcomes in elderly type 2 diabetes patients, many of whom already have coronary heart disease. Evidences from 
clinical and experimental trials have shown that hypoglycemia may cause abnormal electrical cardiac activity. Resulting in ST wave alterations with increasing QT interval and cardiac repolarization [13].

\section{Antidiabetic Therapies and CVD}

Commonly used oral antidiabetic drugs like sulfonylureas and thiazolidinediones may develop certain cardiovascular issues like hypoglycemia-induced ischemia and arrhythmia. In heart tissues, sulfonylureas bind to the channels blocking the following three beneficial mechanisms, 1) vascular smooth muscle cells relaxation that improves coronary blood flow, 2) limitation of myocardial damage during ischemia, and 3) protection of energy producing mitochondria in cardiomyocytes. Monotherapy with 1st and 2nd generation sulfonylureas have been associated with significant higher risk ( $24 \%$ to $61 \%)$ for all-cause mortality while monotherapy with 2 nd generation sulfonylureas were associated with significant excess risk (18\% to $30 \%)$ for congestive heart failure [12].

Thiazolidinediones are known to be associated with occurrence of heart failure due to fluid retention increasing the risk of myocardial infarction. Biguanides are associated with improved cardiovascular outcomes. However, they may induce lactic acidosis and hence should not be used in patients with acute or unstable heart failure. Alpha Glucosidase inhibitors are found to improve CV outcome without any adverse impact by lowering post-prandial glucose excursions especially in carbohydrate eating populations [14].

Chronic hyperinsulinemia is a thought to be atherogenic and thus may offset the benefit conferred by modest glycemic improvement with administration of insulin. Insulin therapy may promote cardiovascular risks like smooth muscle cell proliferations, vasoconstrictions especially in large conduit arteries, monocyte adhesions, fluid retention and prothrombotic activities due to increased level of plasminogen activator receptor-1 (PAI-1), endothelin-1 receptors and adhesion molecules. Hyperinsulinemia in the presence of insulin resistance can overstimulate the intracellular mitogen signaling pathway in endothelial cells. This condition, together with impaired phosphatidylinositol 3-kinase activation of nitric oxide synthase, could lead to an atherogenic state [15].

\section{Glycemic Variability \& CVD}

Glycemic variability is considered not only the 4th pillar of glycemic control but also yet another important determinant for cardiovascular safety. In fact, it is implicated as an independent risk factor for cardiovascular complications in patients with and without diabetes [16]. Post prandial excursion plays an important role in glycemic variability. Although a simple and a standard tool to measure glycemic variability is yet to be established, Mean Amplitude of Glycemic Excursions (MAGE) reduction is documented to lower oxidative stress and inflammatory markers in type 2 diabetic patients and consequently, the cardiovascular risks [17]. Therefore, cardiovascular risk has been found to be reduced 
with antidiabetic drugs with efficacy to maintain glycemic variability at its lowest level.

\section{What Is Cardiovascular Outcome Trial (CVOT)?}

In an endeavor to identify antidiabetic drugs with maximum safety with the least cardiovascular risks, the Food and Drug Administration (FDA) and the European Medicines Agency simultaneously revised their approval processes in 2008, so that all new glucose lowering molecules prove their cardiovascular safety before granting approval. As per these guidelines, an upper limit of the $95 \%$ confidence interval (CI ) for the risk ratio for cardiovascular events of $<1.3$ is recommended as a key criterion for excluding unacceptable CV risk for recent treatment modalities against type 2 diabetes pending approval. Regulatory agencies require enrollment of patients with relatively advanced CV disease, elderly and patients with some degree of renal impairment. The requirements also include $\mathrm{CV}$ safety data with a prospective, independent adjudication of $\mathrm{CV}$ events in all phase 2 and 3 studies over a minimum duration of 2 years. Regulatory agencies demand that adjudication events include CV mortality, acute coronary syndrome and stroke requiring hospitalization, urgent revascularization procedures, and possibly other end points. Moreover, the agencies stipulated new statistical guidelines regarding the analysis of cardiovascular safety data in order to satisfy upper limit of CI with submission of New Drug /Biologic License Application. The accepted methodology consists of meta-analysis of all placebo-controlled trials, add-on trials (drug vs. placebo, each added to standard therapy), prospective randomized placebo controlled trials or an additional single large safety trial alone, or combined with other trials. Most new drugs received approval only after going through this process [18].

\section{Is Insulin Resistance the Main Culprit?}

During follow up of participating subjects for several years after completion of UKPDS, the importance of early aggressive glycemic control in improving CV outcomes was documented (legacy effect). However, the role of moderate to severe insulin resistance was being established in onset of multiple CV risk factors like obesity, dyslipidemia, hypertension, endothelial dysfunction and pro-coagulant state. Therefore, the importance of multifactorial intervention was recognized for improving CV risk factors. Furthermore, the molecular mechanisms responsible for insulin resistance, independent of metabolic abnormalities were documented to directly contribute to pathogenesis of atherosclerosis [19]. Finally, individuals manifesting insulin resistance syndrome without diabetes were noted to present increased risk for CVD similar to patients with T2DM thus supporting the concept that hyperglycemia is not the lone major risk factor for CVD in subjects with diabetes [20]. Therefore, reducing blood pressure to the optimum level and improving the lipid profile leads to greater reduction in CVD risk than lowering plasma glucose alone in T2DM patients. So, conceptually, the anti-diabetes 
agents like insulin or insulin secretagogues (e.g. sulfonylureas, GLP1 receptor agonists and dipeptidyl peptidase 4 (DPP-4) inhibitors) which lower plasma glucose without an effect on insulin resistance, should not lower CV risk and mortality in type 2 diabetes mellitus. However, the data from UKPDS as well as GLP1 RA trials are contradictory to this concept. On the other hand, treatment with metformin, the most effective insulin sensitizer lowered CV outcomes in UKPDS as well [9]. Alternatively, the data regarding glitazones which improve insulin sensitivity is inconsistent. Pioglitazone may have a favorable effect on CV risk in type 2 diabetes mellitus, independent of its glucose-lowering action as suggested in PROactive trial which showed marginal reduction in secondary endpoints alone including MACE (CV death, nonfatal myocardial infarction [MI], nonfatal stroke) by $16 \%(\mathrm{P}<0.027)$ [21]. Role of improving insulin sensitivity in reducing the incidence of recurrent stroke and MI by therapy with pioglitazone in individuals with insulin-resistance without diabetes was evident in IRIS [22]. However, therapy with Rosiglitazone, another insulin sensitizer appeared to increase adverse CV outcomes in subjects with diabetes [23] [24] [25].

\section{Effect of GLP1 RA}

Three point MACE has been found to be reduced by $15 \%$ in a meta-analysis of the two long-acting GLP-1 RAs liraglutide and semaglutide with similar and significant benefit for all three components: nonfatal stroke, nonfatal MI and mortality by $18 \%, 16 \%$, and $13 \%$, respectively. LEADER and SUSTAIN-6 have highlighted that patients at higher cardiovascular risk benefited more from GLP-1 RA treatment. The benefit of liraglutide and semaglutide has been quite visible maximally for optimal control of traditional CV risk factors. However, it is worth noticing the finding in LEADER that $23 \%$ of patients without prior cardiovascular event, did not show MACE reduction. In SUSTAIN-6, there was a greater fall in $\mathrm{HbAlc}$ of the treatment group although hyperglycemia was not considered a major risk factor for cardiovascular disease. There were more incidences of serious eye complications like vitreous hemorrhage, blindness, and photocoagulation [21].

\section{Empa-Reg Outcome Study}

In EMPA-REG OUTCOME, empagliflozin reduced 3 point MACE by $14 \%(\mathrm{P}=$ 0.04 for superiority) in more than seven thousand type 2 diabetes patients with established cardiovascular disease for more than three years [21]. Many outcomes were different from LEADER, SUSTAIN-6, PROactive, and IRIS. The primary outcome was a robust $38 \%$ decrease in $\mathrm{CV}$ mortality $(\mathrm{P}=0.001)$. Surprisingly, the reduction in primary outcome was early at 3 months after starting treatment [21]. However, reduction in all three points of MACE did not match similar patterns. Nonfatal MI decreased only slightly with hazard ratio HR (0.87) which was not statistically significant $(\mathrm{P}=0.22)$. Alternatively, nonfatal stroke HR (1.24) increased slightly but not significantly $(P=0.22)$. 


\section{How Did Our Understanding of Cardiovascular Outcome Change over the Years?}

Insulin resistance is a core defect in type 2 diabetes mellitus which is associated with multiple metabolic and cardiovascular risk factors. The role the molecular physiology of insulin resistance in pathogenesis of atherosclerosis is relatively well documented [19]. Therefore, interventions which improve insulin sensitivity may reduce $\mathrm{CV}$ events in subjects with type 2 diabetes. However, most of the $\mathrm{CV}$ outcome trials except IRIS did not determine insulin resistance or its surrogate markers. It is plausible that improvements in $\mathrm{CV}$ outcomes in clinical trials using GLP-1 RAs, sodium-glucose cotransporter 2 inhibitors (SGLT2 I) and pioglitazone may have been mediated via increase in insulin sensitivity [25] [26]. Pioglitazone is a powerful insulin sensitizer in skeletal muscle, liver, and adipocytes [25]. GLP-1 RAs may increase insulin sensitivity through weight loss, though its major effect is noted in both type 1 and 2 diabetes enhanced insulin secretion, an "incretin" effect. Treatment with dapagliflozin for as little as 2 weeks modestly increases insulin-mediated glucose disposal secondary to reversal of glucotoxicity [26]. However, improvement in cardiovascular outcomes in type 2 diabetes cannot be entirely attributed to lowering insulin resistance since decline in $\mathrm{CV}$ outcomes noted in Extension of diabetes Control Complication Trial (EDIC) in type 1 diabetes was attributed to aggressive glycemic control, "metabolic memory effect" [27]. Moreover, the relationships between the declines in adverse CV outcomes and lowering of $\mathrm{HbA1C}$ in these $\mathrm{CV}$ trials are almost identical when compared to the data in UKPDS and EDIC [10] [28] [29] [30] [31]. Finally, it is likely that almost all manifestations including complications and metabolic abnormalities noted in both type 2 and 1 diabetes are consequences of multicellular dysfunction secondary to lack of entry of glucose, the most efficient fuel caused by insulin resistance and insulin lack respectively [32]. The hypothesis is further established by improvement in manifestations by facilitating glucose entry into cells by amelioration of insulin resistance and relative decline in insulin secretion in type 2 and insulin administration in type 1 diabetes.

The beneficial effect of empagliflozin on cardiovascular events appears to be unique from that of pioglitazone and GLP-1 RAs as robust reduction in CV mortality is rapid in onset, together with a marked decrease in hospitalization for heart failure. Proposed mechanisms for the impressive reductions in both mortality and onset of heart failure may be reductions in "afterload" following rapid simultaneous lowering of blood pressure as well as "preload" and arterial stiffness by declining intravascular volume; i.e. hemodynamic effects and not by slowing the process of atherosclerosis. Alternatively, reduced left ventricle mass index and improved diastolic dysfunction following empagliflozin treatment for 3 months documented in a recent preliminary study may have contributed to improvement in CV outcomes [33]. Increased blood ketone levels, reduced uric acid, and increased angiotensin and angiotensin type 2 receptor activity are also suggested to be responsible for beneficial CV effects in EMPA-REG OUTCOME 
[34]. However, the main contributor to the improvement in heart failure is likely to be diuretic activity of glycosuria [31].

\section{Are These Benfits Additive?}

As the beneficial cardiovascular effects of empagliflozin are hemodynamically mediated and those of GLP-1 RAs and pioglitazone are direct action on the vasculature to reduce atherogenesis, it is plausible that combination therapy with empagliflozin plus pioglitazone and/or a GLP-1 RA may exert an additive, even synergistic, CV benefit. Empagliflozin profoundly reduced CV mortality, whereas pioglitazone and GLP-1 RAs primarily reduced the risk of nonfatal MI and nonfatal stroke, so addition of empagliflozin to pioglitazone or a GLP-1 RA may produce a robust reduction in all three MACE components. However, this hypothesis requires examination.

\section{Summary}

Insulin resistance is the core defect in metabolic syndrome, prediabetes and type 2 diabetes. It initiates endothelial dysfunction before onset of T2DM. It may be an important risk factor for CVD since almost all insulin resistant states e.g. hypoglycemia, aging, obesity, dyslipidemia and hypertension are contributors to CVD morbidity and mortality. Enhancement of insulin sensitivity may be of equal or even greater influence in preventing or delaying CVDs in comparison to attaining and maintaining desirable glycemic control. However the role of hyperglycemia in onset of cardiovascular complications can not be denied. Finally, occurrence of hypoglycemia as a consequence of treatment and various treatment modalities themselves may contribute to increasing cardiovascular risk as well.

\section{Conflicts of Interest}

The author declares no conflicts of interest regarding the publication of this paper.

\section{References}

[1] Matheus, A.S., Tannus, L.R., Cobas, R.A., et al. (2013) Impact of Diabetes on Cardiovascular Disease: An Update. International Journal of Hypertension, 2013, Article ID: 653789. https://doi.org/10.1155/2013/653789

[2] Hsueh, W.A. and Law, R.E. (1998) Cardiovascular Risk Continuum: Implications of Insulin Resistance and Diabetes. American Journal of Medicine, 105, 4S-14S. https://doi.org/10.1016/S0002-9343(98)00205-8

[3] Mohan, V., Shanthirani, C.S., Deepa, M., et al. (2006) Mortality Rates Due to Diabetes in a Selected Urban South Indian Population-The Chennai Urban Population Study [CUPS-16]. Journal of the Association of Physicians of India, 54, 113-117.

[4] Holman, R.R., Paul, S.K., Bethel, M.A., et al. (2008) 10-Year Follow-up of Intensive Glucose Control in Type 2 Diabetes. New England Journal of Medicine, 359, 1577-1589. https://doi.org/10.1056/NEJMoa0806470 
[5] Morrish, N.J., Wang, S.L., Stevens, L.K., Fuller, J.H. and Keen, H. (2001) Mortality and Causes of Death in the WHO Multinational Study of Vascular Disease in Diabetes. Diabetologia, 44, Article No. S14. https://doi.org/10.1007/PL00002934

[6] Action to Control Cardiovascular Risk in Diabetes Study Group (2008) Effects of Intensive Glucose Lowering in Type 2 Diabetes. New England Journal of Medicine, 358, 2545-2559. https://doi.org/10.1056/NEJMoa0802743

[7] ADVANCE Collaborative Group (2008) Intensive Blood Glucose Control and Vascular Outcomes in Patients with Type 2 Diabetes. New England Journal of Medicine, 358, 2560-2572. https://doi.org/10.1056/NEJMoa0802987

[8] Duckworth, W., Abraira, C., Moritz, T., et al. (2009) VADT Investigators. Glucose Control and Vascular Complications in Veterans with Type 2 Diabetes. New England Journal of Medicine, 360, 129-139. https://doi.org/10.1056/NEJMoa0808431

[9] UK Prospective Diabetes Study (UKPDS) Group (1998) Intensive Blood-Glucose Control with Sulphonylureas or Insulin Compared with Conventional Treatment and Risk of Complications in Patients with Type 2 Diabetes (UKPDS 33). Lancet, 352, 837-853. https://doi.org/10.1016/S0140-6736(98)07019-6

[10] Colhoun, H.M., Betteridge, D.J., Durrington, P.N., et al. on Behalf of the CARDS Investigators (2004) Primary Prevention of Cardiovascular Disease with Atorvastatin in Type 2 Diabetes in the Collaborative Atorvastatin Diabetes Study (CARDS): Multicentre Randomised Placebo-Controlled Trial. Lancet, 364, 685-696. https://doi.org/10.1016/S0140-6736(04)16895-5

[11] Heart Outcomes Prevention Evaluation (HOPE) Study Investigators (2000) Effects of Ramipril on Cardiovascular and Microvascular Outcomes in People with Diabetes Mellitus: Results of the HOPE Study and MICRO-HOPE Substudy. Lancet, 355, 253-259. https://doi.org/10.1016/S0140-6736(99)12323-7

[12] Bell, D.S.H. (2006) Do sulfonylurea Drugs Increase the Risk of Cardiac Events? Canadian Medical Association Journal, 174, 185-186. https://doi.org/10.1503/cmaj.051237

[13] Frier, B.M., Schernthaner, G., Heller, S.R. (2011) Hypoglycemia and Cardiovascular Risks. Diabetes Care, 34, S132-S137. https://doi.org/10.2337/dc11-s220

[14] Kaul, S., Bolger, A.F., Herrington, D., et al. (2010) Thiazolidinedione Drugs and Cardiovascular Risks: A Science Advisory from the American Heart Association and American College of Cardiology Foundation. Circulation, 121, 1868-1877. https://doi.org/10.1161/CIR.0b013e3181d34114

[15] Del Prato, S., Bianchi, C., Dardano, A., et al. (2013) Insulin as an Early Treatment for Type 2 Diabetes. Origin or End of an Old Question? Diabetes Care, 36, S198-S204. https://doi.org/10.2337/dcS13-2019

[16] Yu, J.H., Han, K., Park, S., Lee, D.Y., Nam, G.E., Seo, J.A., Kim, S.G., Baik, S.H., Park, Y.G., Kim, S.M., Kim, N.H. and Choi, K.M. (2019) Effects of Long-Term Glycemic Variability on Incident Cardiovascular Disease and Mortality in Subjects without Diabetes. Medicine, 98, Article ID: e16317. https://doi.org/10.1097/MD.0000000000016317

[17] Su, G., Mi, S., Tao, H., et al. (2011) Association of Glycemic Variability and the Presence and Severity of Coronary Artery Disease in Patients with Type 2 Diabetes. Cardiovascular Diabetology, 10, Article No. 19. https://doi.org/10.1186/1475-2840-10-19

[18] Hirshberg, B. and Katz, A. (2013) Cardiovascular Outcome Studies with Novel Antidiabetes Agents: Scientific and Operational Considerations. Diabetes Care, 36, S253-S258. https://doi.org/10.2337/dcS13-2041 
[19] DeFronzo, R.A. (2010) Insulin Resistance, Lipotoxicity, Type 2 Diabetes and Atherosclerosis: The Missing Links. The Claude Bernard Lecture 2009. Diabetologia, 53, 1270-1287. https://doi.org/10.1007/s00125-010-1684-1

[20] Obunai, K., Jani, S. and Dangas, G.D. (2007) Cardiovascular Morbidity and Mortality of the Metabolic Syndrome. Medical Clinics of North America, 91, 1169-1184. https://doi.org/10.1016/j.mcna.2007.06.003

[21] Ghani, M.A., DeFronzo, R.A., Prato, S.D., Chilton, R., Singh, R. and Ryder, R.E.J. (2017) Cardiovascular Disease and Type 2 Diabetes: Has the Dawn of a New Era Arrived? Diabetes Care, 40, 813-820. https://doi.org/10.2337/dc16-2736

[22] Kernan, W.N., Viscoli, C.M., Furie, K.L., et al., for the IRIS Trial Investigators. (2016) Pioglitazone after Ischemic Stroke or Transient Ischemic Attack. New England Journal of Medicine, 374, 1321-1331. https://doi.org/10.1056/NEJMoa1506930

[23] Nissen, S.E. and Wolski, K. (2007) Effect of Rosiglitazone on the Risk of Myocardial Infarction and Death from Cardiovascular Causes. New England Journal of Medicine, 356, 2457-2471. https://doi.org/10.1056/NEJMoa072761

[24] Home, P.D., Pocock, S.J., Beck-Nielsen, H., Curtis, P.S., Gomis, R., Hanefeld, M., Jones, N.P., Komajda, M. and McMurray, J.J.V. (2009) RECORD Study Team Rosiglitazone Evaluated for Cardiovascular Outcomes in Oral Agent Combination Therapy for Type 2 Diabetes (RECORD): A Multicentre, Randomised, Open-Label Trial. Lancet, 373, 2125-2135. https://doi.org/10.1016/S0140-6736(09)60953-3

[25] Eldor, R., DeFronzo, R.A. and Abdul-Ghani, M. (2013) In Vivo Actions of Peroxisome Proliferator-Activated Receptors. Glycemic Control, Insulin Sensitivity, and Insulin Secretion. Diabetes Care, 36, S162-S174. https://doi.org/10.2337/dcS13-2003

[26] Merovci, A., Solis-Herrera, C., Daniele, G., et al. (2014) Dapagliflozin Improves Muscle Insulin Sensitivity but Enhances Endogenous Glucose Production. Journal of Clinical Investigation, 124, 509-514. https://doi.org/10.1172/JCI70704

[27] Nathan, D.M., for the DCCT/EDIC Research Group (2014) The Diabetes Control and Complications Trial/Epidemiology of Diabetes Interventions and Complications Study at 30 Years: Overview. Diabetes Care, 37, 9-16.

https://doi.org/10.2337/dc13-2112

[28] Kabadi, U.M. (2017) Cardiovascular Outcome Trials in Type 2 Diabetes: Reliable or Biased! World Journal of Pharmaceutical and Medical Research 3, 33-35.

[29] Shimazawa, R. and Ikeda, M. (2019) Imbalance in Glycemic Control between the Treatment and Placebo Groups in Cardiovascular Outcome Trials in Type 2 Diabetes. Journal of Pharmaceutical Policy and Practice, 12, Article No. 30. https://doi.org/10.1186/s40545-019-0193-y

[30] Kabadi Udaya, M. (2021) Comparative Effectiveness of Glucose lowering Drugs for Type 2 Diabetes. Annals of Internal Medicine, 174, 140. https://doi.org/10.7326/L20-1277

[31] Kabadi Udaya, M. (2021) Improvement in Cardiovascular and Renal Outcomes in Type 2 Diabetes: Effect of Glycemic Control, not Drugs. Journal of Diabetes, Metabolic Disorders \& Control, 8, 76-77. https://doi.org/10.15406/jdmdc.2021.08.00225

[32] Kabadi, U.M. (2021) Diabetes Mellitus: Disorder of Cellular Dysfunction Due to Lack of Entry into Cell of Glucose. The Most Efficient Fuel for Cellular Function. Open Journal of Endocrine and Metabolic Diseases, 11, 79-101. https://doi.org/10.4236/ojemd.2021.113007

[33] Verma, S., Garg, A., Yan, A.T., et al. (2016) Effect of Empagliflozin on Left Ventricular Mass and Diastolic Function in Individuals with Diabetes: An Important Clue 
to the EMPA-REG OUTCOME Trial? Diabetes Care, 39, e212-e213.

https://doi.org/10.2337/dc16-1312

[34] Abdul-Ghani, M., Del Prato, S., Chilton, R. and DeFronzo, R.A. (2016) SGLT2 Inhibitors and Cardiovascular Risk: Lessons Learned from the EMPA-REG OUTCOME Study. Diabetes Care, 39, 717-725. https://doi.org/10.2337/dc16-0041 